\title{
Erratum: Model-independent bounds on a light Higgs
}

\section{Aleksandr Azatov, Roberto Contino and Jamison Galloway}

Dipartimento di Fisica, Università di Roma "La Sapienza" and INFN Sezione di Roma, I-00185 Rome, Italy

E-mail: aleksandr.azatov@roma1.infn.it, roberto.contino@roma1.infn.it, jamison.galloway@roma1.infn.it

ERRATUM TO: JHEP04(2012)127

The operator $\operatorname{Tr}\left[\left(D_{\mu} \Sigma\right)^{\dagger}\left(D_{\nu} \Sigma\right)\right] \partial^{\mu} \partial^{\nu} F(h)$ is not linearly independent and can be removed by use of equations of motion and integration by parts. On the other hand, the operator $\operatorname{Tr}\left[\left(D_{\mu} \Sigma\right)^{\dagger}\left(D_{\nu} \Sigma\right)\right] \partial^{\mu} F(h) \partial^{\nu} F(h)$ is linearly independent and must be added to the list of bosonic operators in $\mathcal{L}^{4}$. Equation (2.7) should be thus replaced by:

$$
\begin{aligned}
& O_{1}=\operatorname{Tr}\left[\left(D_{\mu} \Sigma\right)^{\dagger}\left(D^{\mu} \Sigma\right)\right]\left(\partial_{\nu} F_{1}(h)\right)^{2} \\
& O_{2}=\operatorname{Tr}\left[\left(D_{\mu} \Sigma\right)^{\dagger}\left(D_{\nu} \Sigma\right)\right] \partial^{\mu} F_{2}(h) \partial^{\nu} F_{2^{\prime}}(h) .
\end{aligned}
$$

\section{Acknowledgments}

We thank Luca Merlo for drawing our attention on the relevance of the operator $\mathrm{O}_{2}$ of eq. (1). 